\title{
Analysis of Diversity and Equalization Techniques Applied to M-QAM Digital Mobile Radio Systems
}

\author{
Antonio Valdovinos, Fernando J. Casadevall \\ Department of Signal Theory and Communications \\ Universidad Politécnica de Cataluña \\ Barcelona, SPAIN
}

\begin{abstract}
This paper presents an investigation of diversity and/or equalization techniques applied to 4 and 16-QAM TDMA radio systems for rapid fading dispersive channels. In particular, Typical Urban (TU) environments have been considered. A Least Sum of Squared Errors (LSSE) channel estimator has been introduced in order to allow the analytic calculation of the equalizer coefficients. By means of simulation methods we have found that a degradation of $4 \mathrm{~dB}$ can be expected in relation to ideal estimation. Furthermore, the degrading effects of non-linear power amplifiers have been analyzed and a degradation of $2 \mathrm{~dB}$ has been found.
\end{abstract}

\section{INTRODUCTION}

In recent years, wireless digital radio systems have been widely studied and much effort has been done to compensate for the harsh multipath fading which affects both indoor and outdoor radio channels. Two major effects of the time varying channel are the intersymbol interference and the Rayleigh fluctuations in the signal-tonoise ratio. In order to combat these effects, a variety of protection techniques must be used. In particular, diversity techniques have proved to be efficient in fighting the Rayleigh fluctuations if the time delay spread is low, [1]. However, when distortion increases, the use of diversity is not enough and the introduction of equalization techniques becomes necessary. Specifically, Decision Feedback Equalizers (DFE) a e more efficient than Linear Transversal Equalizers (LTE) since they can compensate for deeper in-band nulls due to selective fading. Moreover, its performance is close to that of the optimal MLSE-receiver, with the advantage of being considerably less complex. An important point to decide is the way of adjusting the DFE coefficients. The use of RLS algorithms provides good performance only if the doppler spread is very low, but for most channels is too high to achieve an acceptable tracking performance. For high bit rates and not too long bursts it is better to calculate the initial coefficients from the estimated impulse response and to keep them fixed all along the

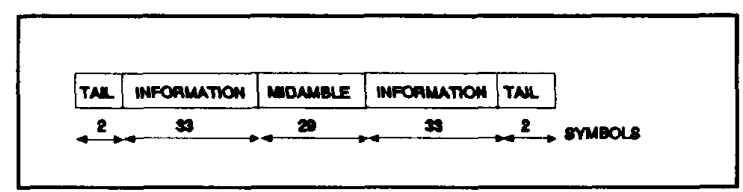

Figure 1. Time slot structure.

burst structure. In order to minimize the fading effects, the burst is designed as shown in fig. 1 , with a midamble instead of a preamble since this way the last symbols of the burst are closer to the point where the impulse response is estimated. The estimator structure is based on the LSSE algorithm presented in [2]. Once the impulse response is estimated and the DFE coefficients calculated, the equalization process is performed in both right and left directions simultaneously.

The system under study has to be efficient in environments where a high density of traffic is generated. Linear modulation schemes such as M-QAM are more spectrum efficient than constant envelope modulation schemes such as GMSK and so, are appropriated to be used in these systems. As a consequence of the envelope variations, the use of high-efficient power amplifiers causes non-linear distortion which introduces an additional degradation in the system performance.

This paper presents the results obtained for 4 and 16QAM radio systems when two receiving antenna and DFE are joint or separately used. The degrading effects due to Rayleigh fluctuations, ISI, doppler spread and non-linear distortion are analyzed by means of computer simulation.

\section{SIMULATION MODEL}

The equivalent complex lowpass representation of the simulated transmission system is shown in fig. 2 .

The M-QAM transmitted signal can be expressed as: 


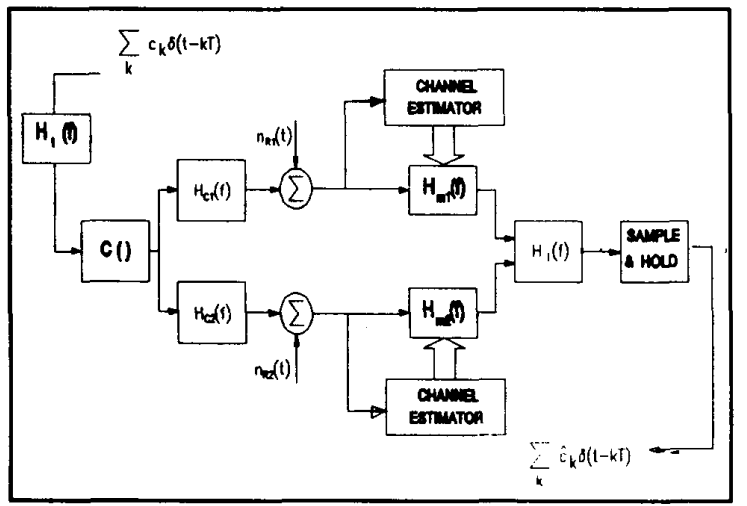

Figure 2. Lowpass equivalent model of the transmission system.

$$
x(t)=\sum_{k=-\infty}^{\infty} a_{k^{\prime}} h_{T}(t-k T)+j b_{k} \cdot h_{T}(t-k T)
$$

where $\left\{a_{k}\right\}$ and $\left\{b_{k}\right\}$ are independent data sequences of duration $T$ for the in-phase and quadrature channels respectively. They are selected from the set $\left\{ \pm 1, \pm 3, \ldots, \pm\left(M^{12}-1\right)\right\}$ with $M=4$ for $4-Q A M$ and $M=16$ for $16-Q A M$. $H_{T}(f)$ is the root-raised cosine filter with a roll-off factor equal to 0.5 and models the transmitted wave form. The complex function $c()$ represents the input-output relationship of the nonlinear power amplifier:

$$
c(|x(t)|)=F(|x(t)|) e^{j \phi(|x(t)|)}
$$

with $|x(t)|$ the module of the input signal. $F(|x(t)|)$ and $\phi(|x(t)|)$ describe the AM/AM and AM/PM conversion effects respectively. We have used an $A B$ class power amplifier given in [3] for which we have fit the AM/AM and AM/PM characteristics by means of the following polinomials:

$$
F(|x(t)|)=|x(t)| \cdot\left(g_{0}+g_{1}|x(t)|+g_{2}\left|x(t)^{\prime}\right|^{2}+g_{3}|x(t)|^{3}\right)(3)
$$

$$
\phi(|x(t)|)=\phi_{x}(t)+p_{o}+p_{1}|x(t)|+p_{2}|x(t)|^{2}+p_{3}|x(t)|^{3}
$$

where $\phi_{x}(t)$ is the phase of the input signal. The polinomial coefficients are adjusted independently for each back-off factor considered.

At the receiver a matched filter $H_{m}(f)$ is incorporated, so, if the power amplifier were perfectly linear, then:

$$
h_{m}(t)=F^{-1}\left[H_{m}(f)\right]=h_{1}^{*}(-t)
$$

where + denotes the conjugate, $\mathrm{F}^{-1}$ is the inverse Fourier transform and $h_{1}(t)$ is the equivalent impulse response prior to $h_{m}(t)$ :

$$
h_{1}(t)=h_{T}(t) * h_{c}(t)
$$

being:

$$
h_{T}(t)=F^{-1}\left[H_{T}(f)\right]
$$

Therefore, the global impulse response results:

$$
h(t)=h_{T}(t) * h_{c}(t) * h_{R}(t)=h_{1}(t) * h_{1}^{*}(-t)=\alpha(t)=\alpha^{+}(-t)(8)
$$

Let us notice as the receiver filter is matched not only to the root-raised cosine transmitting filter but also to the entire impulse response $h_{1}(t)$, which is affected by the channel impulse response variations, and thus needs to be continually updated. It could be implemented in accordance with a FIR structure from the estimated impulse response. The function $h_{c}(t)$ models the channel behavior that introduces selective fading in the radio link. The channel is assumed to be wide-sense stationary uncorrelated scattering (WSSUS) and it is represented by a unique correlation function referred to as the Power Delay Profile, $P(t)$, and given by:

$$
\left\langle h_{c}(t) h_{c}{ }^{*}\left(t^{\prime}\right)\right\rangle=P(t) \delta\left(t-t^{\prime}\right)
$$

where $\langle$. $\rangle$ denotes the ensemble average, and

$$
P(t)=\left\langle\left|h_{c}(t)\right|^{2}\right\rangle
$$

A measure of the width of $P(t)$ is the root meansquare delay spread, $\tau$, defined as:

$$
\tau=\left[\frac{\int\left(t-\tau_{m}\right)^{2} P(t) d t}{\int P(t) d t}\right]^{\frac{1}{2}}
$$

where the average delay $\tau_{\mathrm{m}}$ is defined as:

$$
\tau_{m}=\frac{\int t P(t) d t}{\int P(t) d t}
$$

The multipath phenomenon can be described in terms of time delays with no Doppler shifts for static channels and in terms of time delays and the Doppler shift associated with each delay for the dynamic channels. The model adopted correspond to that proposed by COST207, [4], to describe typical urban (non-hilly) environments and is based on representing the channel by a finite number of taps, each one determined by its time delay and average power. The amplitude of each tap is Rayleigh distributed and varies in accordance with the corresponding Doppler Spectrum. This models is 
described by a continuous power delay profile and approximated through a discrete number of taps.

In absence of non-linear distortion, the received signal for each diversity branch may be expressed as:

$$
\begin{gathered}
r(t)=\sum_{k=-\infty}^{\infty}\left[a_{k} \cdot h^{R}(t-k T)-b_{k} \cdot h^{l}(t-k T)\right]+ \\
+j \sum_{k=-\infty}^{\infty}\left[b_{k} \cdot h^{R}(t-k T)+a_{k} \cdot h^{l}(t-k T)\right]+n_{x}(t)+j n_{y}(t)
\end{gathered}
$$

where

$$
h(t)=h^{R}+j h^{\prime}=\left[F^{-1}\left[H_{T}(f) \cdot H_{m}(f)\right] * h_{c}(t)\right] . G
$$

with * the convolution operator. $G$ is a gain factor that ensures a constant average power at the demodulator input. It is introduced in order to consider the presence of automatic gain control (AGC) and is only necessary for 16-QAM patterns since in 4-QAM we have zerocrossing decision boundaries.

It should be noted here that the carrier and timing recovery circuits are not necessary if the matched filter is present. In this case, $h_{0}$ is real and, therefore, the optimum recovered phase is always zero. In addition, since the maximum of $h(t)$ is in the middle point of this function, the optimum sampling instant is $t_{d}=0$.

The DFE structure with symbol period (T) spacing between stages has been investigated. The minimum mean-square error (MMSE) technique has been adopted to calculate the tap values. Once the impulse channel response is estimated, a set of linear equations can be posed to obtain the optimum tap values. The equations to be solved are different depending on the situation we are dealing with: equalization only, joint equalization and diversity, [1].

\section{RESULTS}

The performance of diversity and/or equalization techniques in mobile rattio channels have been investigated using the bit error rate (BER) as quality criterion. If the system can be described in terms of its global impulse response, that is, if non-linear distortion is not present, an analytical method can be used to compute the error probability integral. In this case, an extension of the Levy method [5] applied to 4 and 16QAM has been considered. When the power amplifier is introduced, the system cannot be described by means of its impulse response and so, it is necessary to appeal to simulation techniques. In particular, a quasi-analytical method has been taken into account for static channels and the traditional Montecarlo method for dynamic ones. In fig.3. the evolution of the BER as a function of the normalized delay spread given by the product $\tau \cdot R_{b}$ for 4QAM is depicted, where $R_{b}$ is the bit rate. The advantage of using this normalization parameter is that we have verified a very similar behavior for the other channel models such as Hilly Terrain (HT), Rural Areas (RA) and bad case for urban (hilly) terrain (BU). Therefore, the results shown here can be extended to these models just considering that the $\tau$ value is different for each one of them (TU: $\tau=1 \mu \mathrm{s}, \mathrm{HT}: \tau=5 \mu \mathrm{s}, \mathrm{RA}: \tau=0.1 \mu \mathrm{s}$, BU: $\tau=2.5 \mu$ ). In this figure we can notice that it is necessary to use some kind of protection technique to compensate for the hostile transmission channel. For low values of the normalized delay spread, the main effect is the signal-to-noise fluctuation due to the Rayleigh fading and not the induced ISI. Thereby, the use of diversity techniques is more appropriate than equalization ones, the improvement being greater than one order of magnitude. However, for $\tau . R_{b}$ higher than 0.1 , the system performance degrades quickly even if diversity is used. In this case the DFE improves the diversity performance, though the BER achieved is still high. It is clear that the best behavior is obtained when joint diversity and DFE techniques are introduced. In this case the receiver must be more complex, but sometimes can be necessary if the transmission quality requires it.

Fig.4. shows the system performance for a static channel as a function of the signal to noise ratio. In particular, a bit rate of $500 \mathrm{Kbit} / \mathrm{s}$, which corresponds to $\tau . R_{b}=0.5$, has been chosen. In this figure a comparison between ideal (continuous line) and real (dashed line) channel estimation is presented. The estimator used is

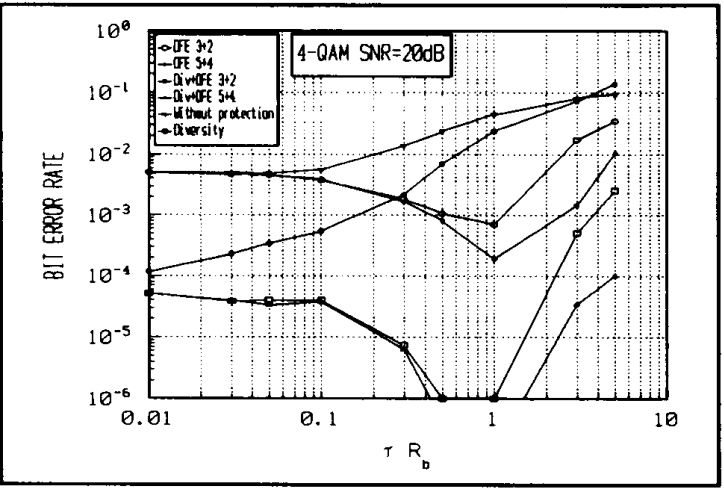

Figure 3. Evolution of the bit error rate (BER) versus the normalized delay spread. 


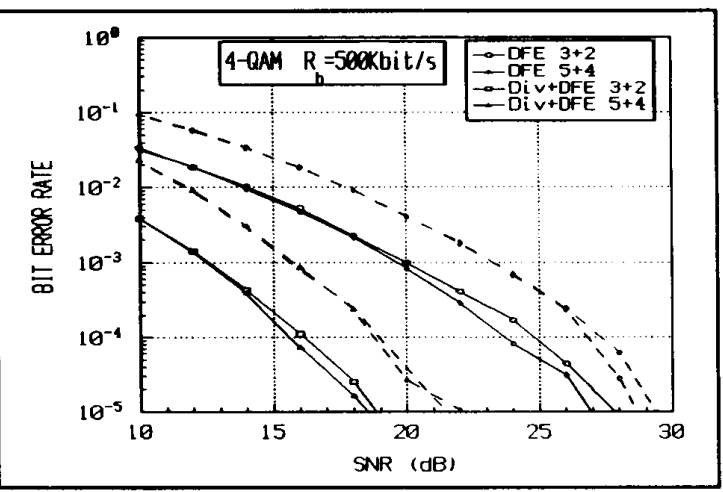

Figure 4. Evolution of the bit error rate (BER) versus the signal-tonoise ratio. Ideal and real estimations in continuous and dashed lines respectively.

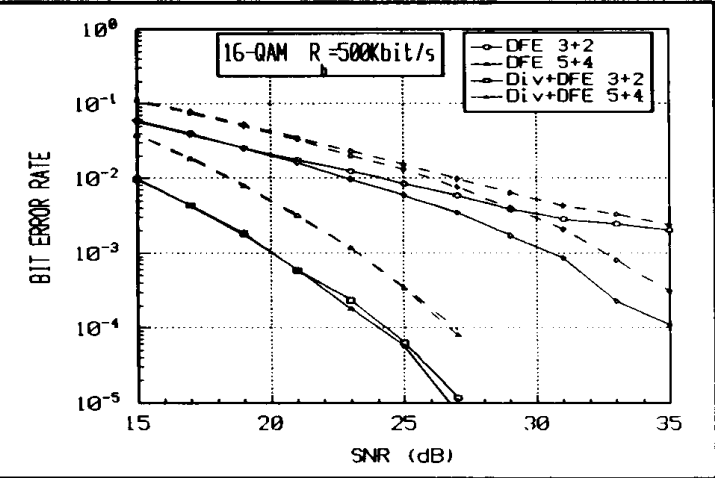

Figure 5. Evolution of the bit error rate (BER) versus the signal-tonoise ratio. Ideal and real estimations in continuous and dashed lines respectively.

able to provide a 10T long estimated impulse response, and it causes a degradation of about $4 \mathrm{~dB}$. In order to improve the estimator performance, it would be necessary to use longer training sequences, with the additional cost in terms of transmission efficiency that this fact represents. In any case, this figure also proves that the use of joint diversity and equalization techniques improve the system performance in more than $8 \mathrm{~dB}$ and it is an efficient tool to combat the degradation induced by the channel.

In order to compare both 4 and 16-QAM patterns, in fig. 5 the BER evolution versus the SNR for 16-QAM is represented. The behavior is similar to that of 4-QAM, but it is clear that the SNR value needed to guarantee a certain value of BER is considerably higher. Actually, a 16-QAM system needs approximately $8 \mathrm{~dB}$ more than 4 QAM to achieve the same performance. Therefore, high-

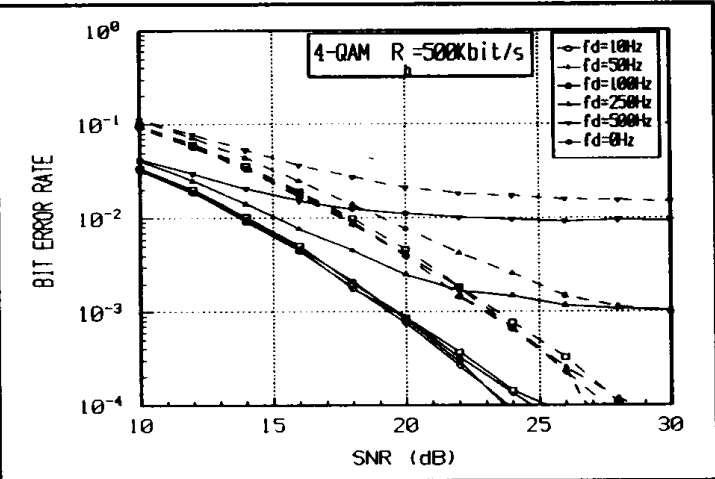

Figure 6. Evolution of the bit error rate (BER) versus the signal-tonoise ratio. Ideal and real estimations in continuous and dashed lines respectively.

level modulation schemes are useful to increase the spectral efficiency, but not the maximum bit rate reached by the system. On the other hand, when a channel estimator is introduced a degradation of about $3 \mathrm{~dB}$ is found, which is almost the same as 4-QAM patterns.

Fig.6 shows the doppler shifts influence over the system performance. It is clear that the system is able to cope with maximum doppler frequency values as high as $100 \mathrm{~Hz}$ without appreciable degradation. In these cases, no tracking is needed, and the matched filter and DFE coefficients can be kept fixed all along the burst. These are usual values for indoor communications or even for outdoor environments if the vehicle speed is not too high (less than about $50 \mathrm{Km} / \mathrm{h}$ ). However, for higher vehicle speeds, the use of some kind of tracking algorithm is needed. In this case the problem is to implement a technique fast enough to follow the channel variations. It seems clear that simple LMS algorithms are not useful in this context and that some Kalman or RLS technique has to be used. This is an important point to investigate since in many cases it can be better not to update the DFE coefficients because of the tracking algorithms instability.

Finally, we have analyzed the system performance when a power amplifier is introduced at the transmitter. Now, as the channel estimator is only able to calculate the linear component of the channel response, the nonlinear distortion induced by the amplifier causes an additional degradation with respect to the linear case which has been found to be approximately $2 \mathrm{~dB}$. Fig. 7 represents the behavior corresponding to a 16-QAM system with an $A B$ class amplifier for different back-off factors referenced with respect to the $1 \mathrm{~dB}$ compression 


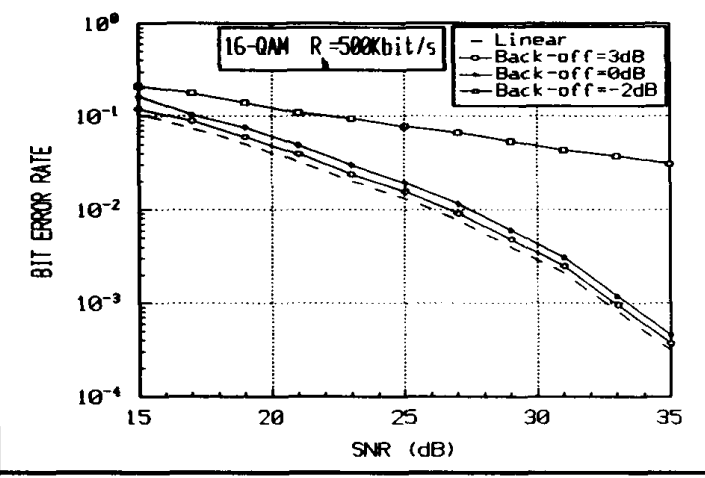

Figure 7. Evolution of the bit error rate (BER) versus the signal-tonoise ratio. Non-linear amplifier.

point (or point defined by the power input at which the amplifier outputs a power $1 \mathrm{~dB}$ below the obtained if the amplifier were exactly linear). From this figure we can conclude that a degradation of about $2 \mathrm{~dB}$ with respect to the linear case could be expected when the amplifier is working at the vicinity of its $1 \mathrm{~dB}$ compression point. Let us notice the important degradation level resulting when the power amplifier working point is over the $1 \mathrm{~dB}$ compression point (negative back-off values).

\section{CONCLUSIONS}

In this paper we have investigated the performance of joint diversity and DFE equalization in a typical mobile environment analyzing some degrading effects due not only to the Rayleigh nature of the dispersive channel but also to the introduction of a realistic channel estimator which provides a finite number of samples of the global impulse response. A degradation value of about 3 or 4 $\mathrm{dB}$ has been found for this particular burst structure. We have also stated the need for using longer training sequences if the transmission quality requires it. Moreover, we have presented a brief comparison between 4 and 16-QAM modulation schemes. From the results obtained we can conclude that the use of high-order QAM patterns does not offer any advantage in terms of maximum bit rate achievable by the system and its use is only advisable when a high spectral efficiency is required. On the other hand, we have considered the use of high-efficient power amplifiers whose input-output characteristic presents non-linear terms. In this case, a degradation of about $2 \mathrm{~dB}$ could be expected in relation to the linear case. Finally, it must be noted that the use of joint diversity and equalization techniques increases considerably the performance achievable by using any of these techniques separately. It must be born in mind that in a practical communication system channel coding and interleaving are introduced and so, the bit error rate can be reduced as much as required.

\section{ACKNOWLEDGEMENTS}

This paper reflects part of the work supported by CICYT (Spain) under Grant TIC 714/90.

\section{REFERENCES}

F.J. Casadevall, A. Valdovinos, "Performance of M-QAM Modulations for Indoor Radio Communications with Combined Diversity and Equalization Techniques". International Joumal of Digital and Analog Communication Systems, vol.5, pp 117-127, April-June 1992.

[2] S.N. Crozier, D.D. Falconer, S.A. Mahmoud, "Least sum of squared errors (LSSE) channel estimation". IEE Proceedings-F, Vol.138, No.4, pp 371-378, August 1991.

[3] S. Ariyavisitakul, "Characterizing the Effects of Nonlinear Amplifiers on Linear Modulation for Digital Portable Radio Communications". IEEE Trans. on Vehicular Technology, Vol.39, No.40, pp 383-389, November 1990

COST 207: "Digital Land Mobile Radio Communications". Final Report. Published by the Commission of the European Communities. Luxembourg, 1989. pp 135-147.

[5] A. Levy, "Fast Error Evaluation in the Presence of Intersymbol interference ", IEEE Trans. on Communications, 1985, COM-33 (5), pp 479-481. 